\title{
Vector profile and gauge invariant observables of string field theory solutions for constant magnetic field background
}

\author{
Nobuyuki Ishibashi, ${ }^{a}$ Isao Kishimoto, ${ }^{b}$ Toru Masuda $^{c, d}$ and Tomohiko Takahashi ${ }^{e}$ \\ ${ }^{a}$ Tomonaga Center for the History of the Universe, \\ University of Tsukuba, Tsukuba, Ibaraki 305-8571, Japan \\ ${ }^{b}$ Faculty of Education, Niigata University, \\ Niigata 950-2181, Japan \\ ${ }^{c}$ CEICO, Institute of Physics of the Czech Academy of Sciences, \\ Na Slovance 1999/2, 18221 Prague 8, Czech Republic \\ ${ }^{d}$ CORE of STEM, Nara Women's University, \\ Nara 630-8506, Japan \\ e Department of Physics, Nara Women's University, \\ Nara 630-8506, Japan \\ E-mail: ishibash@het.ph.tsukuba.ac.jp, ikishimo@ed.niigata-u.ac.jp, \\ masudatoru@gmail.com, tomo@asuka.phys.nara-wu.ac.jp
}

ABSTRACT: We study profiles and gauge invariant observables of classical solutions corresponding to a constant magnetic field on a torus in open string field theory. We numerically find that the profile is not discontinuous on the torus, although the solution describes topologically nontrivial configurations in the context of low energy effective theory. From the gauge invariant observables, we show that the solution provide correct couplings of closed strings to a D-brane with constant magnetic field.

Keywords: Bosonic Strings, String Field Theory, D-branes, Tachyon Condensation ARXIV EPrint: 1804.01284 


\section{Contents}

1 Introduction 1

2 Classical solutions for constant magnetic field background 3

3 Profiles of the classical solution $\quad 4$

3.1 Profiles and dual states 4

3.2 Quasi-periodicity of the solution 5

$\begin{array}{ll}3.3 \text { Tachyon profile } & 7\end{array}$

3.4 Vector profile 8

4 Gauge invariant observables for the classical solution $\quad 11$

$\begin{array}{ll}\text { 4.1 Calculation of gauge invariant observables } & 11\end{array}$

$\begin{array}{ll}4.2 & \text { Comparison with Dirac-Born-Infeld action } \\ & 12\end{array}$

4.3 T-dual of a D2-brane with $F_{12} \neq 0 \quad 13$

5 Concluding remarks $\quad 15$

$\begin{array}{ll}\text { A Conformal transformation of the vector vertex operator } & 16\end{array}$

$\begin{array}{ll}\text { B Calculation of correlation functions in the vector profile } & 17\end{array}$

\section{Introduction}

String field theory is believed to admit classical solutions that describe a wide range of moduli space of string theory. In bosonic open string field theory, Erler and Maccaferri [1] proposed a way to construct classical solutions representing any time-independent open string background by use of boundary condition changing (BCC) operators. Following their method, a solution corresponding to constant magnetic field background has been constructed by some of the present authors in [2]. It was found that the classical action of the solution calculated from the operator product expansions (OPEs) of BCC operators agrees with the Dirac-Born-Infeld action.

This magnetic solution has several new features compared with the solutions discovered so far. Firstly it has no direct relation to tachyon dynamics, such as tachyon condensation or tachyon lump, or it is not obtained simply by marginal deformation of already-known solutions. Secondly, the solution on a torus corresponds to the configuration has a nonvanishing Chern number of the U(1) gauge field. Such a topologically nontrivial solution had never been constructed before in string field theory.

The purpose of this paper is to investigate some physical properties of the magnetic solution. Firstly, we will study the so-called tachyon and vector profiles of the solution 
defined as follows. Using the Fock space expression, a solution $|\Psi\rangle$ of the open string field theory can be expanded as

$$
|\Psi\rangle=\sum_{p} t_{p}\left|T_{\boldsymbol{p}}\right\rangle+\sum_{\boldsymbol{p}} A_{\boldsymbol{p}}{ }^{\mu}\left|V_{\boldsymbol{p}_{\mu}}\right\rangle+\cdots
$$

where the lower mass states, corresponding to the tachyon and the massless vector field are expressed as

$$
\begin{aligned}
\left|T_{\boldsymbol{p}}\right\rangle & =c_{1}|\boldsymbol{p}\rangle=c e^{i \boldsymbol{p} \cdot \boldsymbol{X}}(0)|0\rangle \\
\left|V_{\boldsymbol{p}}{ }^{\mu}\right\rangle & =c_{1} \alpha_{-1}^{\mu}|\boldsymbol{p}\rangle=i \sqrt{\frac{2}{\alpha^{\prime}}} c \partial X^{\mu} e^{i \boldsymbol{p} \cdot \boldsymbol{X}}(0)|0\rangle .
\end{aligned}
$$

The position representation of the component fields is given by the Fourier transform of $t_{\boldsymbol{p}}, A_{\boldsymbol{p}}^{\mu}, \cdots$ :

$$
t(\boldsymbol{x})=\sum_{\boldsymbol{p}} t_{\boldsymbol{p}} e^{i \boldsymbol{p} \cdot \boldsymbol{x}}, \quad A^{\mu}(\boldsymbol{x})=\sum_{\boldsymbol{p}} A_{\boldsymbol{p}}^{\mu} e^{i \boldsymbol{p} \cdot \boldsymbol{x}}, \quad \cdots
$$

$t(\boldsymbol{x})$ and $A^{\mu}(\boldsymbol{x})$ are called the tachyon and vector profiles of the solution respectively.

In conventional field theory, we need to divide the torus into some patches to describe the $\mathrm{U}(1)$ gauge field with a constant magnetic field on a torus, so that the smooth gauge fields on different patches are related by gauge transformations. Therefore it is natural to ask whether the magnetic solution needs multiple patches in string field theory. This question may be examined by evaluating the vector profile $A^{\mu}(\boldsymbol{x})$ corresponding to the $\mathrm{U}(1)$ gauge field. If $A^{\mu}(\boldsymbol{x})$ has discontinuities, we need to divide the torus by coordinate patches to represent the configuration by smooth gauge fields.

Secondly, we will evaluate gauge invariant observables for the magnetic solution. In conventional field theory, topologically non-trivial configurations are characterized by some gauge invariant quantities which take discrete values. Such quantities have not been found in string field theory. Instead, we have the gauge invariant observable which is associated with on-shell closed string vertex operators [3]:

$$
O_{V}(\Psi) \equiv\langle I|c(i) c(-i) V(i,-i)| \Psi\rangle
$$

where $I$ is the identity string field, $V(z, \bar{z})$ denotes an on-shell closed string vertex operator and $z=+i, \bar{z}=-i$ in the complex plane correspond to the midpoint $\sigma=\pi / 2$ of the open string. If $\Psi$ is a classical solution, the observable represents a coupling of the closed string vertex operator $V$ to the D-brane to which the solution corresponds. Accordingly, we expect that the observable for a massless anti-symmetric tensor vertex has a non-trivial value since the corresponding D-brane has constant background magnetic field. In order to confirm the existence of background magnetic field and find a clue for topological invariants in string field theory, we calculate the gauge invariant observables with massless and zeromomentum closed string vertex operators.

This paper is organized as follows. In section 2 , we briefly review the constant magnetic solution on torus. In section 3 , we study tachyon and vector profiles of the solution. As 
a by-product, we show periodic and quasi-periodic properties of the solutions. In section 4, we evaluate the gauge invariant observables of the solution. By comparing the resulting observables with the Dirac-Born-Infeld (DBI) action, we find that the solution indeed corresponds to constant magnetic field background. In section 5, we will give concluding remarks. In the appendices, details of calculations are exhibited.

\section{Classical solutions for constant magnetic field background}

We would like to consider the configuration with a constant background $F_{\mu \nu}$. We concentrate on the spatial directions $X^{1}$ and $X^{2}$, since a real antisymmetric tensor $F_{\mu \nu}$ can be transformed into a block diagonal form with $2 \times 2$ blocks. Let us consider the bosonic open string field theory in which these spatial directions are toroidally compactified with radii $R_{1}$ and $R_{2}$, and the Neumann boundary conditions are imposed on the variables $X^{1}, X^{2}$. The time direction $X^{0}$ is required to be noncompact in order to construct the solution following Erler-Maccaferri's method [1], but other directions are unspecified here.

To find the classical solution corresponding to a constant $F_{12}$ background, we need to prepare the BCC operators which changes the open string boundary conditions for $X^{1}, X^{2}$ from the Neumann boundary condition to the one with $F_{12}$ and vice versa. Such operators correspond to the open strings with one edge with the free boundary conditions and the other coupled to the constant magnetic field. The zero mode coordinates $x^{1}, x^{2}$ of these open strings become noncommutative and we need to introduce the following operators

$$
U=\exp \left(i \frac{x^{1}}{R_{1}}\right), \quad V=\exp \left(i \frac{x^{2}}{R_{2}}\right)
$$

which satisfy

$$
U V=e^{i \frac{2 \pi}{N}} V U
$$

where the integer $N$ is related to the magnetic field through the Dirac quantization condition:

$$
(2 \pi)^{2} R_{1} R_{2} F_{12}=2 \pi N
$$

The zero mode algebra has a $|N|$ dimensional representation. Correspondingly, we can find $|N|$ pairs of BCC operators: $\sigma_{*}^{k}, \bar{\sigma}_{*}^{k}(k=1, \cdots,|N|)[2]$, which are primary fields with conformal weight

$$
h=\frac{\lambda(1-\lambda)}{2}, \quad\left(\tan \pi \lambda=2 \pi \alpha^{\prime} F_{12}\right) .
$$

Following Erler-Maccaferri's method, we multiply $\sigma_{*}^{k}, \bar{\sigma}_{*}^{k}$ by the vertex operators $e^{ \pm i \sqrt{h} X^{0}}$ and appropriate normalization factors and construct $|N|$ pairs of modified BCC operators $\sigma^{k}, \bar{\sigma}^{k}$. They are primary fields with conformal weight zero and satisfy the OPEs

$$
\bar{\sigma}^{k}(s) \sigma^{l}(0) \sim \delta_{k, l}, \quad \sigma^{l}(s) \bar{\sigma}^{k}(0) \sim \frac{\delta_{l, k}}{|\cos \pi \lambda|}=\frac{\delta_{l, k}}{\sqrt{1+\left(2 \pi \alpha^{\prime} F_{12}\right)^{2}}},
$$

for small positive $s$. 
Having these BCC operators, the classical solution corresponding to the constant magnetic field background [2] are given as follows:

$$
\Psi_{0}=\Psi_{\mathrm{tv}}+\sum_{k, l} A_{k, l} \Phi^{k, l}
$$

where $\Psi_{\mathrm{tv}}$ is the Erler-Schnabl solution for the tachyon vacuum [4],

$$
\Phi^{k, l}=-\frac{1}{\sqrt{1+K}} c(1+K) \sigma^{k} \frac{B}{1+K} \bar{\sigma}^{l}(1+K) c \frac{1}{\sqrt{1+K}},
$$

and $A_{k, l}$ is a hermitian $|N| \times|N|$ matrix satisfying $A^{2}=A$. The second term is a solution to the equations of motion around the tachyon vacuum. Suppose that $A$ is given by

$$
A=\operatorname{diag}(\underbrace{1, \cdots, 1}_{M}, \underbrace{0, \cdots, 0}_{|N|-M})
$$

then the solution can be regarded as describing $M$ D-branes with magnetic field condensation. Using the OPEs (2.3), it is easy to show that the energy of the solution is given by $M$ times that of a single D-brane with magnetic field condensation.

For later discussion, it is convenient to rewrite the solution (2.4) in the following way. By using the algebra of $K, B, c$ and $\sigma^{k}, \bar{\sigma}^{l}$, the solution $\Phi^{k, l}$ can be decomposed to three parts:

$$
\Phi^{k, l}=-\frac{\delta_{k, l}}{|\cos \pi \lambda|} \Psi_{\mathrm{tv}}+\phi_{1}^{k, l}+Q_{\mathrm{B}} \phi_{2}^{k, l}
$$

where $\phi_{1}^{k, l}$ and $\phi_{2}^{k, l}$ are defined as

$$
\begin{aligned}
\phi_{1}^{k, l} & =-\frac{1}{2} \frac{1}{\sqrt{1+K}} c \partial \sigma^{k} \frac{1}{1+K} \bar{\sigma}^{l} \frac{1}{\sqrt{1+K}}+\frac{1}{2} \frac{1}{\sqrt{1+K}} \sigma^{k} \frac{1}{1+K} \partial \bar{\sigma}^{l} c \frac{1}{\sqrt{1+K}}, \\
\phi_{2}^{k, l} & =\frac{1}{2} \frac{1}{\sqrt{1+K}} c \partial \sigma^{k} \frac{B}{1+K} \bar{\sigma}^{l} \frac{1}{\sqrt{1+K}}+\frac{1}{2} \frac{1}{\sqrt{1+K}} \sigma^{k} \frac{B}{1+K} \partial \bar{\sigma}^{l} c \frac{1}{\sqrt{1+K}} .
\end{aligned}
$$

It should be noted that $\phi_{1}^{k, l}$ and $\phi_{2}^{k, l}$ independently satisfy the string field reality condition, ${ }^{1}$

$$
\left\{\sum_{k, l} A_{k, l} \phi_{1}^{k, l}\right\}^{\ddagger}=\sum_{k, l} A_{k, l} \phi_{1}^{k, l}, \quad\left\{\sum_{k, l} A_{k, l} Q_{\mathrm{B}} \phi_{2}^{k, l}\right\}^{\ddagger}=\sum_{k, l} A_{k, l} Q_{\mathrm{B}} \phi_{2}^{k, l} .
$$

These are useful in checking the correctness of the profile calculation presented later.

\section{Profiles of the classical solution}

\subsection{Profiles and dual states}

Now let us study the profiles of the magnetic solution $\Psi_{0}$. In order to extract momentum space profiles from the solution expanded as (1.1), we define the states dual to the tachyon

\footnotetext{
${ }^{1}$ We follow the definition of the conjugate $\ddagger$ given in [1]. K, B and $c$ are self-conjugate and $\sigma^{\ddagger}=\bar{\sigma}$. In addition, we find $(\partial \sigma)^{\ddagger}=-\partial \bar{\sigma}$.
} 
and massless vector states, $\left|T_{\boldsymbol{p}}\right\rangle$ and $\left|V_{\boldsymbol{p}_{\mu}}\right\rangle$, by

$$
\begin{aligned}
& \left|\tilde{T}_{\boldsymbol{p}}\right\rangle=\frac{1}{(2 \pi)^{2} R_{1} R_{2}} c_{0} c_{1}|-\boldsymbol{p}\rangle=-\frac{1}{(2 \pi)^{2} R_{1} R_{2}} c \partial c e^{-i \boldsymbol{p} \cdot \boldsymbol{X}}(0)|0\rangle, \\
& \left|\tilde{V}_{\boldsymbol{p}}^{\mu}\right\rangle=\frac{1}{(2 \pi)^{2} R_{1} R_{2}} c_{0} c_{1} \alpha_{-1}^{\mu}|-\boldsymbol{p}\rangle=-\frac{1}{(2 \pi)^{2} R_{1} R_{2}} i \sqrt{\frac{2}{\alpha^{\prime}}} c \partial c \partial X^{\mu} e^{-i \boldsymbol{p} \cdot \boldsymbol{X}}(0)|0\rangle .
\end{aligned}
$$

These are dual to $\left|T_{\boldsymbol{p}}\right\rangle$ and $\left|V_{\boldsymbol{p}_{\mu}}\right\rangle$ in the sense that they satisfy

$$
\left\langle\tilde{T}_{\boldsymbol{p}}, T_{\boldsymbol{q}}\right\rangle=\delta_{\boldsymbol{p}, \boldsymbol{q}}, \quad\left\langle\tilde{V}_{\boldsymbol{p}}^{\mu}, V_{\boldsymbol{q}}^{\nu}\right\rangle=\delta_{\boldsymbol{p}, \boldsymbol{q}} \eta^{\mu \nu}, \quad\left\langle\tilde{T}_{\boldsymbol{p}}, V_{\boldsymbol{q}}^{\mu}\right\rangle=\left\langle\tilde{V}_{\boldsymbol{p}}^{\mu}, T_{\boldsymbol{q}}\right\rangle=0,
$$

and they are orthogonal to other higher massive states. The momentum space profile is derived from the inner product of the dual state and $\Psi_{0}$ :

$$
t_{\boldsymbol{p}}=\left\langle\tilde{T}_{\boldsymbol{p}}, \Psi_{0}\right\rangle, \quad A_{\boldsymbol{p}}{ }^{\mu}=\left\langle\tilde{V}_{\boldsymbol{p}}^{\mu}, \Psi_{0}\right\rangle .
$$

Using (2.7), we can see that the profiles (3.4) are decomposed to three parts. The calculations are simplified by using the following identities:

$$
\begin{aligned}
Q_{\mathrm{B}}\left|\tilde{T}_{\boldsymbol{p}}\right\rangle & =0, \\
Q_{\mathrm{B}}\left|\tilde{V}_{\boldsymbol{p}}^{\mu}\right\rangle & =-\frac{1}{(2 \pi)^{2} R_{1} R_{2}} i \sqrt{\frac{2}{\alpha^{\prime}}} \frac{i}{2} \alpha^{\prime} p^{\mu} c \partial c \partial^{2} c e^{-i \boldsymbol{p} \cdot \boldsymbol{X}}(0)|0\rangle .
\end{aligned}
$$

Consequently, for example, we only need to deal with $\Psi_{\mathrm{tv}}$ and $\phi_{1}^{k, l}$ for the calculation of the tachyon profile.

\subsection{Quasi-periodicity of the solution}

Before starting the calculation of the profiles, we would like to point out that the profiles satisfy quasi-periodic relations. Here we deal with the solution corresponding to a single D-brane with constant magnetic field $F_{12} \neq 0$, namely $M=1$ in (2.6). We can construct $|N|$ independent solutions

$$
\Psi_{0}^{k}=\Psi_{\mathrm{tv}}+\Phi^{k, k} \quad(k=1, \cdots,|N|) .
$$

To derive the tachyon profile from $\Psi_{0}^{k}$, we have to calculate the inner products $\left\langle\tilde{T}_{\boldsymbol{p}}, \Psi_{\mathrm{tv}}\right\rangle$ and $\left\langle\tilde{T}_{\boldsymbol{p}}, \phi_{1}^{k, k}\right\rangle$ as seen from (3.5). The former has been known to be a constant [4]. ${ }^{2}$ The latter can be calculated by rewriting in terms of the correlation function including the tachyon vertex and the BCC operators, which has been derived in [2]:

$$
\begin{aligned}
\left\langle e^{-i \boldsymbol{p} \cdot \boldsymbol{X}}(\xi) \sigma_{*}^{k}\left(\xi_{1}\right) \bar{\sigma}_{*}^{l}\left(\xi_{2}\right)\right\rangle & =\frac{\left(\xi_{1}-\xi_{2}\right)^{\alpha^{\prime} \boldsymbol{p}^{2}-\lambda(1-\lambda)}}{\left(\xi-\xi_{1}\right)^{\prime} \boldsymbol{p}^{2}\left(\xi-\xi_{2}\right)^{\alpha^{\prime} \boldsymbol{p}^{2}}} C_{-\boldsymbol{p}}^{l, k}, \\
C_{-\boldsymbol{p}}^{l, k} & =\omega^{\frac{n_{1} n_{2}}{2}-n_{2} l} \delta_{k-l,-n_{1}(\bmod N)} \delta^{-\frac{\alpha^{\prime} \boldsymbol{p}^{2}}{2}},
\end{aligned}
$$

$$
\left\langle\tilde{T}_{\boldsymbol{p}}, \Psi_{\mathrm{tv}}\right\rangle=\int_{0}^{\infty} d t \int_{0}^{\infty} d s \frac{e^{-s-t}}{4 \pi^{2} \sqrt{t s}}(s+t+1)^{2}\left(1+\cos \frac{\pi(t-s)}{s+t+1}\right)=0.509038 \cdots
$$


where $\omega$ is an $N$-th root of unity, $\omega=\exp (2 \pi i / N)$, and $n_{i}$ are momentum quantum numbers, $p_{i}=n_{i} / R_{i} \quad(i=1,2)$, and $\ln \delta=2 \psi(1)-\psi(\lambda)-\psi(1-\lambda)$ for the digamma function $\psi(x)$. Here, it is important to notice that the correlation function depends on the parameters $k$ and $l$ only through the normalization factor, $C_{-p}^{k, l}$. Given this, we find that the inner product $\left\langle\tilde{T}_{\boldsymbol{p}}, \phi_{1}^{k, k}\right\rangle$ depends on $k$ as follows,

$$
\left\langle\tilde{T}_{\boldsymbol{p}}, \phi_{1}^{k, k}\right\rangle=C_{-\boldsymbol{p}}^{k, k} \times \cdots=e^{-i \frac{2 \pi R_{2} k}{N} p^{2}} \times \cdots
$$

Therefore, it turns out that the tachyon profile $t^{k}(\boldsymbol{x})$ of $\Psi_{0}^{k}$ satisfies the quasi-periodic relation:

$$
t^{k}\left(x^{1}, x^{2}+\frac{2 \pi R_{2}}{N}\right)=t^{k-1}\left(x^{1}, x^{2}\right) .
$$

Moreover, since the factor $C_{-\boldsymbol{p}}^{k, k}$ includes $\delta_{0,-n_{1}(\bmod N)}$ we find

$$
t^{k}\left(x^{1}+\frac{2 \pi R_{1}}{N}, x^{2}\right)=t^{k}\left(x^{1}, x^{2}\right)
$$

Similarly to the tachyon case, other profiles can be calculated also by using a 3-point functions of the BCC operators. The matter vertex operators are of the form $\partial^{n} X \cdots e^{i p X}$. Since the operators $U, V$ are not included in the derivatives of $X^{1}, X^{2}$ [2], the 3-point function depends on the parameters $k$ and $l$ only through $C_{-p}^{k, l}$. Consequently, we find that all profiles of the solution satisfy the quasi-periodic relations similar to (3.11) and (3.12), namely, the space representation of the solution satisfies

$$
\begin{aligned}
& \Psi_{0}^{k}\left(x^{1}+\frac{2 \pi R_{1}}{N}, x^{2}\right)=\Psi_{0}^{k}\left(x^{1}, x^{2}\right) \\
& \Psi_{0}^{k}\left(x^{1}, x^{2}+\frac{2 \pi R_{2}}{N}\right)=\Psi_{0}^{k-1}\left(x^{1}, x^{2}\right) .
\end{aligned}
$$

Notice that the quasi-periodicity relations of these forms arise because we have taken the BCC operators corresponding to the eigenstates of $V=\exp \left(i x^{2} / R_{2}\right)$ [2]. If we take the BCC operators corresponding to the eigenstates of $U=\exp \left(i x^{1} / R_{1}\right)$, we have a set of classical solutions which is periodic in the $x^{1}$ direction and quasi-periodic in the $x^{2}$ direction. In addition, we should comment that, due to the translational symmetry of the theory, we can generate other set of solutions from $\Psi_{0}^{k}$ by arbitrary displacement of the torus. In our case, we simply just choose $\Psi_{0}^{k}$ as $|N|$ independent solutions in the sense that they correspond to degenerate states in magnetic fields, so-called Landau level. ${ }^{3}$

\footnotetext{
${ }^{3}$ In a naive expectation, the $|N|$ independent solutions $\Psi_{0}^{k}(k=1, \cdots,|N|)$ are physically equivalent as a result of the translational symmetry and the relation (3.14). However, it is difficult to connect the solutions to each other by a gauge transformation, because we suffer from associativity anomalies [6] if the translation is represented as a gauge transformation in open string field theory.
} 


\subsection{Tachyon profile}

Now let us calculate the tachyon profile. The inner product $\left\langle\tilde{T}_{\boldsymbol{p}}, \phi_{1}^{k, l}\right\rangle$ is rewritten in terms of correlation functions with the help of $K B c$ algebra:

$$
\begin{aligned}
\left\langle\tilde{T}_{\boldsymbol{p}}, \phi_{1}^{k, l}\right\rangle= & \left(\frac{2}{\pi}\right)^{\alpha^{\prime} \boldsymbol{p}^{2}-1} \frac{1}{(2 \pi)^{2} R_{1} R_{2}} \frac{1}{2} \int_{0}^{\infty} \frac{d t_{3}}{\sqrt{\pi t_{3}}} \int_{0}^{\infty} d t_{2} \int_{0}^{\infty} \frac{d t_{1}}{\sqrt{\pi t_{1}}} e^{-t_{1}-t_{2}-t_{3}} \times \\
& \times\left\{\left\langle e^{-i \boldsymbol{p} \cdot \boldsymbol{X}}\left(L-\frac{1}{2}\right) \partial \sigma^{k}\left(t_{1}+t_{2}\right) \bar{\sigma}^{l}\left(t_{1}\right)\right\rangle_{L}\left\langle c \partial c\left(L-\frac{1}{2}\right) c\left(t_{1}+t_{2}\right)\right\rangle_{L}\right. \\
& \left.-\left\langle e^{-i \boldsymbol{p} \cdot \boldsymbol{X}}\left(L-\frac{1}{2}\right) \sigma^{k}\left(t_{1}+t_{2}\right) \partial \bar{\sigma}^{l}\left(t_{1}\right)\right\rangle_{L}\left\langle c \partial c\left(L-\frac{1}{2}\right) c\left(t_{1}\right)\right\rangle_{L}\right\},
\end{aligned}
$$

where $L=t_{1}+t_{2}+t_{3}+1$ and $\langle\cdots\rangle_{L}$ denotes a correlation function on the infinite cylinder of circumference $L$. The correlation functions which appear in (3.15) can be obtained by conformally transforming (3.8) and the ghost correlation functions which are defined on the complex plane. We eventually get the tachyon profile for the $|N|$-th solution $\Psi_{0}^{|N|}$ as

$$
\begin{aligned}
t(\boldsymbol{x})= & \left(1-\frac{1}{|\cos \pi \lambda|}\right) t_{0} \\
& +\frac{2}{|\cos \pi \lambda|}\left[\sum_{m=1}^{\infty} G_{t}\left(\left(\frac{N m}{R_{1}}\right)^{2}\right) \cos \frac{N m x^{1}}{R_{1}}+\sum_{n=1}^{\infty} G_{t}\left(\left(\frac{n}{R_{2}}\right)^{2}\right) \cos \frac{n x^{2}}{R_{2}}\right. \\
& \left.\left.+2 \sum_{n, m=1}^{\infty} G_{t}\left(\left(\frac{N m}{R_{1}}\right)^{2}+\left(\frac{n}{R_{2}}\right)^{2}\right)\right)(-1)^{m n} \cos \frac{N m x^{1}}{R_{1}} \cos \frac{n x^{2}}{R_{2}}\right]
\end{aligned}
$$

where $t_{0}=\left\langle\tilde{T}_{\boldsymbol{p}}, \Psi_{\mathrm{tv}}\right\rangle$. The function $G_{t}(u)$ is defined by

$$
\begin{aligned}
G_{t}(u) & =-\frac{\alpha^{\prime} u}{4} g\left(\alpha^{\prime} u-1\right) \exp \left(-\frac{\alpha^{\prime} u}{2}(2 \psi(1)-\psi(\lambda)-\psi(1-\lambda)-2 \log 4)\right), \\
g(z) & =\int_{0}^{\infty} d t \int_{0}^{\infty} d s \int_{s+t+1}^{\infty} d L \frac{e^{1-L}}{\pi \sqrt{t s}}\left(\frac{\sin \frac{\pi(s+t+1)}{L}}{2 L \sin \frac{\pi(s+1 / 2)}{L} \sin \frac{\pi(t+1 / 2)}{L}}\right)^{z} .
\end{aligned}
$$

The tachyon profile for the $k$-th solution can be derived by using (3.11).

Now, we carry out numerical evaluation of the tachyon profile. In order to do so, we need to evaluate the triple integration on the right hand side of (3.18). However, this expression is inappropriate for numerical integration, because the rate of convergence is very slow due to the infinite integration region and it gives an inaccurate value even by use of Monte Carlo method. An expression of $g(z)$ which is better suited than (3.18) for numerical integration can be obtained by a change of variables:

$$
\begin{aligned}
g(z)= & \frac{2}{\pi} \int_{0}^{1} d v \int_{0}^{1-v} d w \int_{0}^{\frac{\pi}{2}} d \theta e^{1-\frac{1}{v}} v^{z-3} \times \\
& \times\left(\frac{\sin \pi w}{2 \sin \pi\left(\frac{v}{2}+(1-w-v) \sin ^{2} \theta\right) \sin \pi\left(\frac{v}{2}+(1-w-v) \cos ^{2} \theta\right)}\right)^{z} .
\end{aligned}
$$

The plot of $g(z)$ obtained by numerically evaluating it using this expression is depicted in figure 1 . We find that the function rapidly decreases as we increase $z$. The discontinuity of the profile depends on the behavior of $g(z)$ when $z$ is very large. 


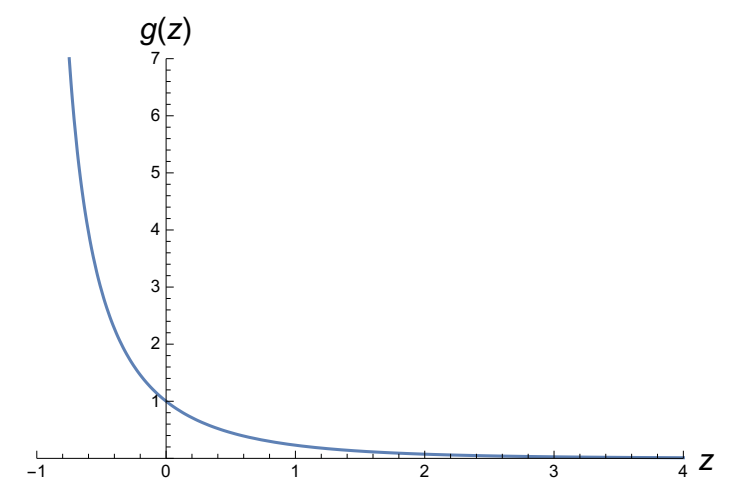

Figure 1. The plots of $g(z)$.

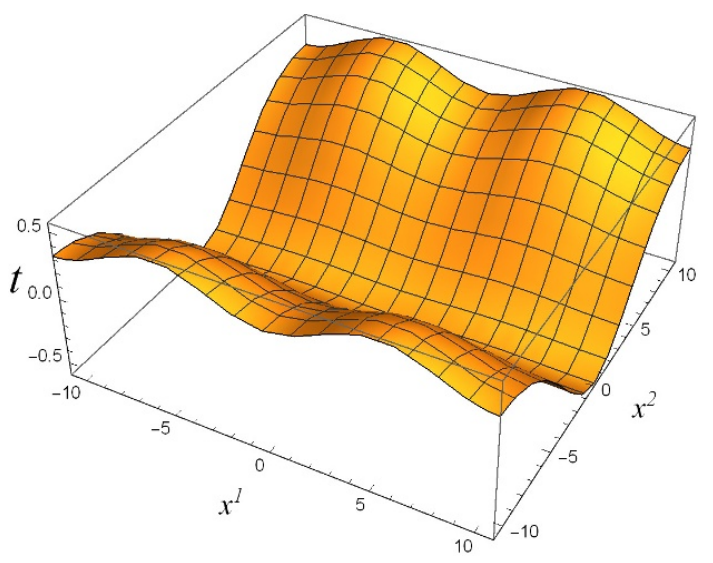

Figure 2. The numerical plots of the tachyon profile in the case of $N=2$ and $R_{1}=R_{2}=2 \sqrt{3}$. Here, we take $\alpha^{\prime}=1$.

The Fourier coefficients of (3.16) are evaluated by using the numerical results of $g(z)$ and the tachyon profile can be obtained numerically by summing up the Fourier series. We find that the Fourier coefficient approaches zero fast enough as $n, m \rightarrow \infty$ so that we can approximate the series by a finite summation over lower modes. The plot of a result is depicted in figure $2 .{ }^{4}$

\subsection{Vector profile}

Next, we consider the vector profile of the magnetic solution. Since the vector profile of $\Psi_{\mathrm{tv}}$ vanishes, the momentum space vector profile of $\Psi_{0}^{k}$ is given by

$$
A_{\boldsymbol{p}}{ }^{\mu}=\left\langle\tilde{V}_{\boldsymbol{p}}^{\mu}, \phi_{1}^{k, k}\right\rangle-\left\langle Q_{\mathrm{B}} \tilde{V}_{\boldsymbol{p}}^{\mu}, \phi_{2}^{k, k}\right\rangle .
$$

As in the case of the tachyon profile, each inner product can be rewritten in terms of correlation functions on infinite cylinder of circumference $L=t_{1}+t_{2}+t_{3}+1$ :

$$
\left\langle\tilde{V}_{\boldsymbol{p}}^{\mu}, \phi_{1}^{k, l}\right\rangle=\left(\frac{2}{\pi}\right)^{\alpha^{\prime} \boldsymbol{p}^{2}} \frac{1}{(2 \pi)^{2} R_{1} R_{2}} i \sqrt{\frac{2}{\alpha^{\prime}}} \frac{1}{2} \int_{0}^{\infty} \frac{d t_{3}}{\sqrt{\pi t_{3}}} \int_{0}^{\infty} d t_{2} \int_{0}^{\infty} \frac{d t_{1}}{\sqrt{\pi t_{1}}} e^{-t_{1}-t_{2}-t_{3}}
$$

\footnotetext{
${ }^{4}$ The reason why the $x^{1}$ and $x^{2}$ dependence are different from each other is that the solution is constructed by using the BCC operators corresponding to the eigenstates of $V=\exp \left(i x^{2} / R_{2}\right)$.
} 


$$
\begin{aligned}
& \times\left\{\left\langle\partial X^{\mu} e^{-i \boldsymbol{p} \cdot \boldsymbol{X}}\left(L-\frac{1}{2}\right) \partial \sigma^{k}\left(t_{1}+t_{2}\right) \bar{\sigma}^{l}\left(t_{1}\right)\right\rangle_{L}\left\langle c \partial c\left(L-\frac{1}{2}\right) c\left(t_{1}+t_{2}\right)\right\rangle_{L}\right. \\
&\left.-\left\langle\partial X^{\mu} e^{-i \boldsymbol{p} \cdot \boldsymbol{X}}\left(L-\frac{1}{2}\right) \sigma^{k}\left(t_{1}+t_{2}\right) \partial \bar{\sigma}^{l}\left(t_{1}\right)\right\rangle_{L}\left\langle c \partial c\left(L-\frac{1}{2}\right) c\left(t_{1}\right)\right\rangle_{L}\right\}, \quad(3.21) \\
&\left\langle Q_{\mathrm{B}} \tilde{V}_{\boldsymbol{p}}^{\mu}, \phi_{2}^{k, l}\right\rangle=-\left(\frac{2}{\pi}\right)^{\alpha^{\prime} \boldsymbol{p}^{2}} \frac{1}{(2 \pi)^{2} R_{1} R_{2}} i \sqrt{\frac{\alpha^{\prime}}{2}} i p^{\mu} \frac{1}{2} \int_{0}^{\infty} \frac{d t_{3}}{\sqrt{\pi t_{3}}} \int_{0}^{\infty} d t_{2} \int_{0}^{\infty} \frac{d t_{1}}{\sqrt{\pi t_{1}}} e^{-t_{1}-t_{2}-t_{3}} \\
& \times\left\{\left\langle e^{-i \boldsymbol{p} \cdot \boldsymbol{X}}\left(L-\frac{1}{2}\right) \partial \sigma^{k}\left(t_{1}+t_{2}\right) \bar{\sigma}^{l}\left(t_{1}\right)\right\rangle_{L}\left\langle c \partial c \partial^{2} c\left(L-\frac{1}{2}\right) c\left(t_{1}+t_{2}\right) B\right\rangle_{L}\right. \\
&\left.+\left\langle e^{-i \boldsymbol{p} \cdot \boldsymbol{X}}\left(L-\frac{1}{2}\right) \sigma^{k}\left(t_{1}+t_{2}\right) \partial \bar{\sigma}^{l}\left(t_{1}\right)\right\rangle_{L}\left\langle c \partial c \partial^{2} c\left(L-\frac{1}{2}\right) B c\left(t_{1}\right)\right\rangle_{L}\right\},
\end{aligned}
$$

where we have used the expressions for the dual vector vertex operator, (3.2) and (3.6).

The computation of the correlation functions on the right hand side of (3.21) is not so simple because the vector vertex operator in (3.21) is not a primary field. As derived in appendix A, under the conformal transformation $z=f(\xi)$, the vector vertex transforms as follows;

$$
\partial X^{\mu} e^{-i \boldsymbol{p} \cdot \boldsymbol{X}}(z)=\left(\frac{d \xi}{d z}\right)^{\alpha^{\prime} \boldsymbol{p}^{2}+1}\left\{\partial X^{\mu} e^{-i \boldsymbol{p} \cdot \boldsymbol{X}}(\xi)+\frac{i}{2} \alpha^{\prime} p^{\mu} \frac{\frac{d^{2} \xi}{d z^{2}}}{\left(\frac{d \xi}{d z}\right)^{2}} e^{-i \boldsymbol{p} \cdot \boldsymbol{X}}(\xi)\right\} .
$$

Thus, we need correlation functions for vector and tachyon vertex operators to derive the vector profile. A detailed derivation of these correlation functions is presented in appendix B. Using the results, it turns out that only the term (3.21) contributes and we find:

$$
\begin{aligned}
A_{\boldsymbol{p}}{ }^{\mu}= & \frac{\lambda-\frac{1}{2}}{|\cos \pi \lambda|} \sqrt{2 \alpha^{\prime}} i \epsilon^{\mu \nu} p_{\nu} C_{-\boldsymbol{p}}^{k, k} \times \\
& \times(-h) \int_{0}^{\infty} \frac{d t_{3}}{\sqrt{\pi t_{3}}} \int_{0}^{\infty} d t_{2} \int_{0}^{\infty} \frac{d t_{1}}{\sqrt{\pi t_{1}}} e^{-L+1}\left(\frac{2}{L} \frac{\sin \theta_{t_{2}}}{\sin \theta_{t_{1}+\frac{1}{2}} \sin \theta_{t_{3}+\frac{1}{2}}}\right)^{h-1},
\end{aligned}
$$

where $h=\alpha^{\prime} \boldsymbol{p}^{2}+1$ and $\theta_{s}$ is defined by $\theta_{s}=\pi s / L$. By the Fourier transformation of (3.24), the position space representation of the vector profile is obtained as follows:

$$
\begin{aligned}
A_{1}(\boldsymbol{x})= & \frac{\lambda-\frac{1}{2}}{|\cos \pi \lambda|} 2 \sqrt{2 \alpha^{\prime}}\left[\sum_{n=1}^{\infty} \frac{n}{R_{2}} G\left(\left(\frac{n}{R_{2}}\right)^{2}\right) \sin \frac{n x^{2}}{R_{2}}\right. \\
& \left.+2 \sum_{n, m=1}^{\infty} \frac{n}{R_{2}} G\left(\left(\frac{N m}{R_{1}}\right)^{2}+\left(\frac{n}{R_{2}}\right)^{2}\right)(-1)^{m n} \cos \frac{N m x^{1}}{R_{1}} \sin \frac{n x^{2}}{R_{2}}\right], \\
A_{2}(\boldsymbol{x})= & -\frac{\lambda-\frac{1}{2}}{|\cos \pi \lambda|} 2 \sqrt{2 \alpha^{\prime}}\left[\sum_{n=1}^{\infty} \frac{N m}{R_{1}} G\left(\left(\frac{n}{R_{2}}\right)^{2}\right) \sin \frac{N m x^{1}}{R_{1}}\right. \\
& \left.+2 \sum_{n, m=1}^{\infty} \frac{N m}{R_{1}} G\left(\left(\frac{N m}{R_{1}}\right)^{2}+\left(\frac{n}{R_{2}}\right)^{2}\right)(-1)^{m n} \sin \frac{N m x^{1}}{R_{1}} \cos \frac{n x^{2}}{R_{2}}\right],
\end{aligned}
$$

where the function $G(u)$ is defined in terms of $g(z)$ in (3.18) as

$$
G(u)=\left(\alpha^{\prime} u+1\right) g\left(\alpha^{\prime} u\right) \exp \left(-\frac{\alpha^{\prime} u}{2}(2 \psi(1)-\psi(\lambda)-\psi(1-\lambda)-2 \log 4)\right) .
$$




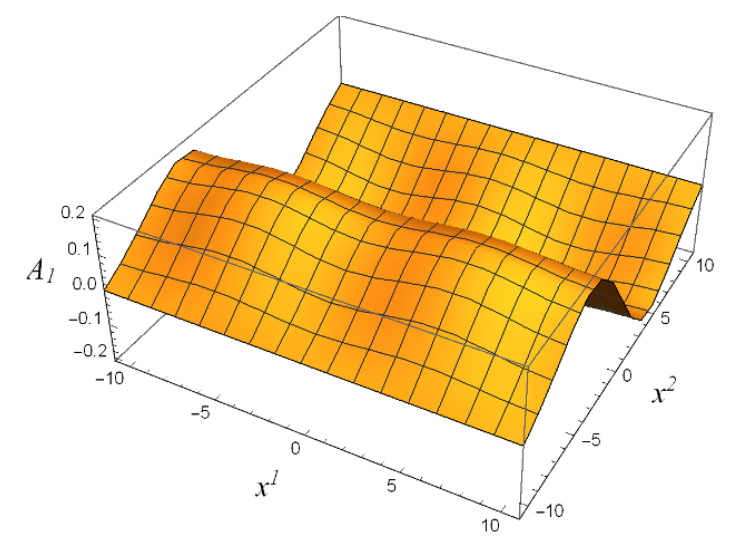

Figure 3. The numerical plots of the vector profile of $A_{1}(\boldsymbol{x})$ in the case of $N=2$ and $R_{1}=R_{2}=$ $2 \sqrt{3}$. Here, we take $\alpha^{\prime}=1$.

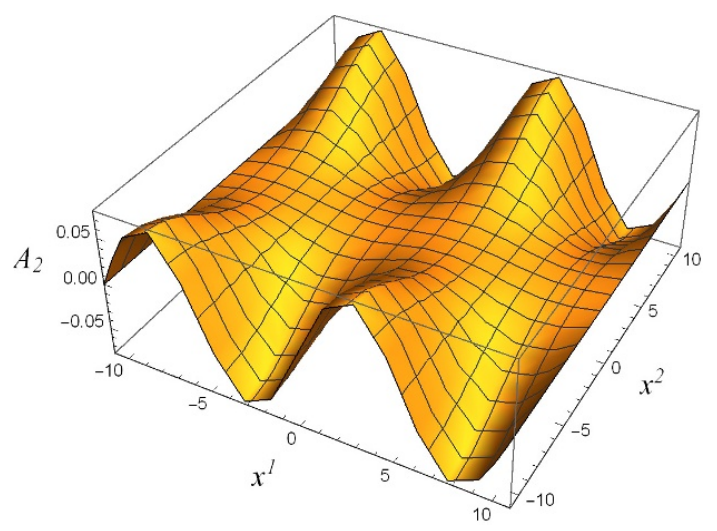

Figure 4. The numerical plots of the vector profile of $A_{2}(\boldsymbol{x})$ in the case of $N=2$ and $R_{1}=R_{2}=$ $2 \sqrt{3}$. Here, we take $\alpha^{\prime}=1$.

As in the tachyon profile, we can numerically calculate the vector profile by using the numerical results of $g(z)$ and summing up the Fourier series. The difference of the two profiles is in the fact that the Fourier coefficients of the vector profile include momentum factors, $N m / R_{1}$ or $n / R_{2}$. Hence the asymptotic behavior of the profile may change in the ultraviolet region and discontinuities could be found for the vector profile.

However, we observe that the Fourier coefficients for the vector profile rapidly converges to zero as $n, m \rightarrow \infty$ and the position space representation of the profile seems to be absolutely convergent. Consequently, we get the plot shown in figures 3 and 4 , and we conclude that the vector profile has no discontinuities. ${ }^{5}$

Here, we would like to comment on profiles for other massive modes. The reason why the tachyon and vector profiles are not discontinuous is because the momentum space profile becomes zero rapidly for large momenta. This behavior is due to the exponential factor

\footnotetext{
${ }^{5}$ From the vector profile, we can calculate the quantity $\tilde{F}_{12} \equiv \partial_{1} A_{2}-\partial_{2} A_{1}$. The resulting $\tilde{F}_{12}$ is given not as a constant but as a smooth function, and the space average of $\tilde{F}_{12}$ over the torus becomes zero. Since $\tilde{F}_{12}$ is not invariant under gauge transformations in string field theory, it is no wonder that $\tilde{F}_{12}$ does not correspond to the constant magnetic field.
} 
$\delta^{-\alpha^{\prime} \boldsymbol{p}^{2} / 2}$ in the normalization factor of the 3 point function (3.9). ${ }^{6}$ Since this exponential factor always appears in the expression of other profiles, we expect that other profiles also do not have any discontinuities.

\section{Gauge invariant observables for the classical solution}

\subsection{Calculation of gauge invariant observables}

Let us consider the gauge invariant observable (1.5) for the $k$-th solution (3.7) with the following closed string vertex operators at zero momentum:

$$
\begin{aligned}
B(z, \bar{z}) & \equiv i(\partial X \bar{\partial} \tilde{X}-\partial \tilde{X} \bar{\partial} X)(z, \bar{z}), \\
G(z, \bar{z}) & \equiv(\partial X \bar{\partial} \tilde{X}+\partial \tilde{X} \bar{\partial} X)(z, \bar{z}),
\end{aligned}
$$

where $X=\left(X^{1}+i X^{2}\right) / \sqrt{2}$ and $\tilde{X}=\left(X^{1}-i X^{2}\right) / \sqrt{2}$. These correspond to the antisymmetric tensor field $B_{12}$ and the sum of graviton field $G_{11}+G_{22}$ in the spatial directions $X^{1}$ and $X^{2}$. Substituting the $k$-th solution (3.7) into (1.5) and expressing it in terms of the correlation functions on the infinite cylinder, the observables can be rewritten as

$$
\begin{aligned}
& O_{V}\left(\Psi_{0}^{k}\right)=\left(1-\frac{1}{|\cos \pi \lambda|}\right) O_{V}\left(\Psi_{\mathrm{tv}}\right) \\
&-\int_{0}^{\infty} \frac{d t_{1}}{\sqrt{\pi t_{1}}} \int_{0}^{\infty} d s \int_{0}^{\infty} \frac{d t_{2}}{\sqrt{\pi t_{2}}} e^{-s-t_{1}-t_{2}} \\
& \times\left\langle V\left(s+t_{1}+i \infty, s+t_{1}-i \infty\right) \partial \sigma^{k}(s) \bar{\sigma}^{k}(0)\right\rangle_{C_{L}} \\
& \times\left\langle c\left(s+t_{1}+i \infty\right) c\left(s+t_{1}-i \infty\right) c(s)\right\rangle_{C_{L}},
\end{aligned}
$$

where $L=s+t_{1}+t_{2}$. For $B(z, \bar{z})$ and $G(z, \bar{z}), O_{V}\left(\Psi_{\mathrm{tv}}\right)$ has been calculated as ${ }^{7}$

$$
\begin{aligned}
& O_{B}\left(\Psi_{\mathrm{tv}}\right)=0, \\
& O_{G}\left(\Psi_{\mathrm{tv}}\right)=-\frac{\alpha^{\prime}}{2 \pi i} \times(2 \pi)^{2} R_{1} R_{2} .
\end{aligned}
$$

Here we normalize the observable by dividing it by the volume of the directions other than $X^{1}$ and $X^{2}$.

For the vertex operators (4.1) and (4.2), the matter correlation function in (4.3) can be derived from the correlator

$$
\left\langle\bar{\sigma}^{k}|\partial X(z) \bar{\partial} \tilde{X}(\bar{w})| \sigma^{l}\right\rangle=-\frac{\alpha^{\prime}}{2} \frac{\lambda\left(\frac{z}{\bar{w}}\right)^{1-\lambda}+(1-\lambda)\left(\frac{\bar{w}}{z}\right)^{\lambda}}{(z-\bar{w})^{2}} \times\left\langle\bar{\sigma}^{k} \mid \sigma^{l}\right\rangle,
$$

\footnotetext{
${ }^{6}$ It gives exponential factor in (3.17) and $(3.27)$, where $2 \psi(1)-\psi(\lambda)-\psi(1-\lambda)-2 \log 4 \geq 0$ for $0 \leq \lambda<1$.

${ }^{7}$ As in [7], if $V(z, \bar{z})$ is decomposed by the matter primary field $V_{n}(z)$ as $V(z, \bar{z})=\sum_{m, n} \zeta_{m n} V_{m}(z) V_{n}(\bar{z})$, and the OPE of the primary fields is $V_{m}(z) V_{n}\left(z^{\prime}\right) \sim v_{m n} /\left(z-z^{\prime}\right)^{2}$, the observable for the tachyon vacuum solutions is given by

$$
O_{V}\left(\Psi_{\mathrm{tv}}\right)=\frac{1}{2 \pi i} \sum_{m, n} \zeta_{m n} v_{m n} \times{ }_{\mathrm{mat}}\langle 0 \mid 0\rangle_{\mathrm{mat}}
$$
}


and its conjugate. Here $\left\langle\bar{\sigma}^{k} \mid \sigma^{l}\right\rangle$ is given by

$$
\left\langle\bar{\sigma}^{k} \mid \sigma^{l}\right\rangle=\frac{(2 \pi)^{2} R_{1} R_{2}}{|\cos \pi \lambda|} \delta_{k, l}
$$

The matter 4-point function in (4.3) on the infinite cylinder can be obtained from (4.6) by a conformal transformation. To make our calculation well-defined, we regularize the correlators by replacing $\pm i \infty$ by $\pm i M$ and taking the limit $M \rightarrow \infty$. As a result, we find

$$
\begin{aligned}
& \left\langle\partial X(x+i M) \bar{\partial} \tilde{X}(x-i M) \sigma^{k}\left(x_{1}\right) \bar{\sigma}^{k}\left(x_{2}\right)\right\rangle_{C_{L}} \\
\rightarrow & -\frac{\alpha^{\prime}}{2}\left(\frac{\pi}{L}\right)^{2}(-4) e^{-\frac{\pi}{L} 4 M}\left\{\lambda e^{i \frac{2 \pi}{L}(1-\lambda)\left(x_{1}-x_{2}\right)}+(1-\lambda) e^{-i \frac{2 \pi}{L} \lambda\left(x_{1}-x_{2}\right)}\right\} \times \frac{(2 \pi)^{2} R_{1} R_{2}}{|\cos \pi \lambda|} .
\end{aligned}
$$

Multiplying the ghost correlator, the 4-point function in (4.3) is given as

$$
\begin{gathered}
\left\langle\partial X\left(s+t_{1}+i \infty\right) \bar{\partial} \tilde{X}\left(s+t_{1}-i \infty\right) \partial \sigma^{k}(s) \bar{\sigma}^{k}(0)\right\rangle_{C_{L}}\left\langle c\left(s+t_{1}+i \infty\right) c\left(s+t_{1}-i \infty\right) c(s)\right\rangle_{C_{L}} \\
=\frac{\alpha^{\prime}}{2} \lambda(1-\lambda)\left\{e^{-i \frac{2 \pi}{L} \lambda s}-e^{i \frac{2 \pi}{L}(1-\lambda) s}\right\} \times \frac{(2 \pi)^{2} R_{1} R_{2}}{|\cos \pi \lambda|}
\end{gathered}
$$

where $L=s+t_{1}+t_{2}$. Similarly its conjugate is given by

$$
\begin{gathered}
\left\langle\bar{\partial} X\left(s+t_{1}-i \infty\right) \partial \tilde{X}\left(s+t_{1}+i \infty\right) \partial \sigma^{k}(s) \bar{\sigma}^{k}(0)\right\rangle_{C_{L}}\left\langle c\left(s+t_{1}+i \infty\right) c\left(s+t_{1}-i \infty\right) c(s)\right\rangle_{C_{L}} \\
=\frac{\alpha^{\prime}}{2} \lambda(1-\lambda)\left\{e^{-i \frac{2 \pi}{L}(1-\lambda) s}-e^{i \frac{2 \pi}{L} \lambda s}\right\} \times \frac{(2 \pi)^{2} R_{1} R_{2}}{|\cos \pi \lambda|}
\end{gathered}
$$

which is also obtained by acting the $X^{2}$ parity transformation $\lambda \leftrightarrow 1-\lambda$ on (4.9) [2].

Having calculated the correlation functions, the observables can be obtained by integration of (4.3):

$$
\begin{aligned}
O_{B}\left(\Psi_{0}\right)= & \frac{\alpha^{\prime}}{\pi i} \times(2 \pi)^{2} R_{1} R_{2} \times \frac{2 \pi \alpha^{\prime} F_{12}}{\sqrt{1+\left(2 \pi \alpha^{\prime} F_{12}\right)^{2}}} \\
O_{G}\left(\Psi_{0}\right)= & \frac{i \alpha^{\prime}}{2 \pi}(2 \pi)^{2} R_{1} R_{2} \\
& \times\left\{1-\sqrt{1+\left(2 \pi \alpha^{\prime} F_{12}\right)^{2}}+2\left(\sqrt{1+\left(2 \pi \alpha^{\prime} F_{12}\right)^{2}}-\frac{1}{\sqrt{1+\left(2 \pi \alpha^{\prime} F_{12}\right)^{2}}}\right)\right\} .
\end{aligned}
$$

\subsection{Comparison with Dirac-Born-Infeld action}

Let us check if the gauge invariant observables (4.11) and (4.12) are consistent with what is known about the magnetic background. Here we consider a D2-brane extended in the $X^{1}$ and $X^{2}$ directions for simplicity. The coupling of the D2-brane to massless modes of closed strings is described by the Dirac-Born-Infeld (DBI) action: ${ }^{8}$

$$
S=-T_{2} e^{-\Phi}(2 \pi)^{2} R_{1} R_{2} \sqrt{\operatorname{det}\left(G_{a b}+B_{a b}+2 \pi \alpha^{\prime} F_{a b}\right)},
$$

\footnotetext{
${ }^{8}$ Hereafter, we adopt the static gauge and normalize the action by dividing it by the volume of the time direction.
} 
where $T_{2}$ is the D2-brane tension, and $\Phi, G_{a b}$ and $B_{a b}$ denote the induced fields on the brane.

Fixing to the static gauge, we find the variation of the DBI action under an infinitesimal variation of $B_{12}$ around the flat background, namely $\left\langle G_{a b}\right\rangle=\delta_{a b}$ and $\left\langle B_{a b}\right\rangle=0$ :

$$
\delta S=-T_{2} e^{-\Phi}(2 \pi)^{2} R_{1} R_{2} \frac{2 \pi \alpha^{\prime} F_{12}}{\sqrt{1+\left(2 \pi \alpha^{\prime} F_{12}\right)^{2}}} \delta B_{12}
$$

The observable (4.11) can be expressed by using the DBI action as

$$
O_{B}\left(\Psi_{0}\right)=\frac{i \alpha^{\prime}}{\pi T_{2} e^{-\Phi}}\left\{\left.\frac{\delta S}{\delta B_{12}}\right|_{F_{12} \neq 0}-\left.\frac{\delta S}{\delta B_{12}}\right|_{F_{12}=0}\right\} .
$$

Similarly, we find the variation of the DBI action under an infinitesimal variation of $G_{a b}$ :

$$
\begin{aligned}
\delta S= & -T_{2} e^{-\Phi}(2 \pi)^{2} R_{1} R_{2} \\
& \times \frac{1}{2}\left\{\frac{1}{2} \sqrt{1+\left(2 \pi \alpha^{\prime} F_{12}\right)^{2}}-\sqrt{1+\left(2 \pi \alpha^{\prime} F_{12}\right)^{2}}+\frac{1}{\sqrt{1+\left(2 \pi \alpha^{\prime} F_{12}\right)^{2}}}\right\}\left(\delta G_{11}+\delta G_{22}\right) .
\end{aligned}
$$

Notice that the variation $\delta G_{12}$ does not appear in this result and the graviton field is included implicitly in the dilaton field as $\Phi=\Phi_{24}+(1 / 4) \ln \operatorname{det} G_{a b}$ when the space-time is of the form $M^{24} \times T^{2}$ [8]. From (4.16) we get the relation

$$
O_{G}\left(\Psi_{0}\right)=\frac{i \alpha^{\prime}}{\pi T_{2} e^{-\Phi}}\left\{\left.\left(\frac{\delta S}{\delta G_{11}}+\frac{\delta S}{\delta G_{22}}\right)\right|_{F_{12} \neq 0}-\left.\left(\frac{\delta S}{\delta G_{11}}+\frac{\delta S}{\delta G_{22}}\right)\right|_{F_{12}=0}\right\} .
$$

These results (4.15) and (4.17) show that the observables correctly reflect the coupling of the D2-brane with the constant magnetic field to the closed string modes. Consequently, it is explicitly found that (3.7) can be regarded as the classical solution corresponding to magnetic field condensation.

\subsection{T-dual of a D2-brane with $F_{12} \neq 0$}

We would like to examine if the couplings (4.11) and (4.12) are consistent with T-duality. It is well known that the D2-brane is transformed into a D-string tilted in the dual torus, which is extended along the line [8]

$$
X^{1}=2 \pi \alpha^{\prime} F_{12} X^{2}+\text { const. }
$$

where $X^{\prime 1}$ denotes the coordinate dual to $X^{1}$. From the Dirac quantization condition (2.1), it follows that the D-string winds $N$ times around the $X^{2}$ direction.

The DBI action for the D-string is given by

$$
S=-T_{1} \int d \xi e^{-\Phi^{\prime}} \sqrt{\operatorname{det}\left[G_{a b}^{\prime}+B_{a b}^{\prime}+2 \pi \alpha^{\prime} F_{a b}\right]}
$$


where $T_{1}$ is the D-string tension and $G_{a b}^{\prime}, B_{a b}^{\prime}$ and $\Phi^{\prime}$ are the induced fields in the dual space-time. Here, we take the coordinate $\xi$ on the D-string to coincide with $X^{2}$. Then, the embedding function $\phi(\xi)$ is given by

$$
\phi^{1}(\xi)=2 \pi \alpha^{\prime} F_{12} X^{2}+\text { const. }, \quad \phi^{2}(\xi)=X^{2} .
$$

In this gauge, the induced metric is calculated as

$$
G_{11}^{\prime}(\xi)=G_{M N}^{\prime} \frac{\partial \phi^{M}}{\partial \xi} \frac{\partial \phi^{N}}{\partial \xi}=\left(2 \pi \alpha^{\prime} F_{12}\right)^{2} G_{11}^{\prime}+4 \pi \alpha^{\prime} F_{12} G_{12}^{\prime}+G_{22}^{\prime} .
$$

Substituting this into the DBI action (4.19), we can calculate the variation of $S$ under an infinitesimal variation of $G_{a b}^{\prime}$ :

$$
\begin{aligned}
\delta S= & -T_{1} e^{-\Phi^{\prime}} 2 \pi R_{2} \\
& \times\left\{-\frac{1}{4} \sqrt{1+\left(2 \pi \alpha^{\prime} F_{12}\right)^{2}}\left(\delta G_{11}^{\prime}+\delta G_{22}^{\prime}\right)\right. \\
& \left.+\frac{1}{2} \frac{1}{\sqrt{1+\left(2 \pi \alpha^{\prime} F_{12}\right)^{2}}}\left\{\left(2 \pi \alpha^{\prime} F_{12}\right)^{2} \delta G_{11}^{\prime}+4 \pi \alpha^{\prime} F_{12} \delta G_{12}^{\prime}+\delta G_{22}^{\prime}\right\}\right\},
\end{aligned}
$$

where we have used the relation $\Phi^{\prime}=\Phi_{24}^{\prime}-(1 / 4) \ln \operatorname{det} G_{a b}^{\prime}$ as in the D2-brane case.

By using the relations between the parameters [8]

$$
e^{-\Phi^{\prime}}=\frac{R_{1}}{\sqrt{\alpha^{\prime}}} e^{-\Phi}, \quad T_{1}=2 \pi \sqrt{\alpha^{\prime}} T_{2},
$$

$\delta S$ can be rewritten as

$$
\begin{aligned}
\delta S= & -T_{2} e^{-\Phi}(2 \pi)^{2} R_{1} R_{2} \\
& \times\left\{\frac{2 \pi \alpha^{\prime} F_{12}}{\sqrt{1+\left(2 \pi \alpha^{\prime} F_{12}\right)^{2}}} \delta G_{12}^{\prime}\right. \\
& +\left(-\frac{1}{4} \sqrt{1+\left(2 \pi \alpha^{\prime} F_{12}\right)^{2}}+\frac{1}{2} \frac{1}{\sqrt{1+\left(2 \pi \alpha^{\prime} F_{12}\right)^{2}}}\right)\left(-\delta G_{11}^{\prime}\right) \\
& \left.+\left(-\frac{1}{4} \sqrt{1+\left(2 \pi \alpha^{\prime} F_{12}\right)^{2}}+\frac{1}{2} \frac{1}{\sqrt{1+\left(2 \pi \alpha^{\prime} F_{12}\right)^{2}}}\right) \delta G_{22}^{\prime}\right\} .
\end{aligned}
$$

Combining with the results (4.11) and (4.12), we find that

$$
\begin{aligned}
& O_{B}\left(\Psi_{0}\right)=\frac{i \alpha^{\prime}}{\pi T_{2} e^{-\Phi}}\left\{\left.\frac{\delta S}{\delta G_{12}^{\prime}}\right|_{F_{12} \neq 0}-\left.\frac{\delta S}{\delta G_{12}^{\prime}}\right|_{F_{12}=0}\right\} \\
& O_{G}\left(\Psi_{0}\right)=\frac{i \alpha^{\prime}}{\pi T_{2} e^{-\Phi}}\left\{\left.\left(\frac{\delta S}{\delta\left(-G_{11}^{\prime}\right)}+\frac{\delta S}{\delta G_{22}^{\prime}}\right)\right|_{F_{12} \neq 0}-\left.\left(\frac{\delta S}{\delta\left(-G_{11}^{\prime}\right)}+\frac{\delta S}{\delta G_{22}^{\prime}}\right)\right|_{F_{12}=0}\right\}
\end{aligned}
$$

These relations implies that the gauge invariant observables reproduce correct couplings of the D-sting to the closed string modes in the dual space, because, in the dual space, the vertex operators (4.1) and (4.2) correspond to $G_{12}^{\prime}$ and a $-G_{11}^{\prime}+G_{22}^{\prime}$ respectively. 


\section{Concluding remarks}

In this paper, we have studied and explicitly calculated the tachyon and vector profiles for the constant magnetic field solution on a torus constructed by following Erler-Maccaferri's method. In addition, we have calculated gauge invariant observables for the solution and found that the solution reproduces correct couplings of the D-brane with a constant magnetic field to the closed string modes.

A remarkable feature of the resulting profiles is that they have no discontinuity on the torus. This result does not seem to be consistent with the fact that the solution corresponds to a topologically nontrivial configuration. In low energy field theory, the gauge field will have discontinuities if one tries to describe the configuration without dividing the torus into patches. We expect that the same thing happens in string field theory, but we have found that profiles for any states do not have discontinuities. One possibility is that profiles for normalized states are not the right quantities to be chosen in observing such phenomena. Instead, we might have to consider a sum of infinitely many profiles or even a nonlinear functional of the string field. Another possibility is that our results could be an indication of nonlocality of string field theory. Since the star product is a nonlocal operation from the target space viewpoint, even if a string field is defined in a coordinate patch, it can spread beyond the boundary after a gauge transformation. Therefore, the non-existence of discontinuity itself may be a natural consequence of the nonlocality of string field theory.

Another important question about the solution is how we can define the topological invariant characterizing the constant magnetic field solution in the framework of string field theory. The magnetic field is proportional to an integer due to the Dirac quantization condition, which is derived based on low energy theory. This quantization condition should be derived from the string field theory itself. Unfortunately, the gauge invariant observables calculated in this paper do not provide any clue about such a quantization condition, although it is interesting to notice that the observable with the antisymmetric tensor mode becomes non-zero as a result of the magnetic field background. In order to capture the "topological" nature, we may need new insights from noncommutative geometry, matrix theory and so on [9-12].

There are many interesting possibilities for future exploration related to the constant magnetic field solution. Suppose that we consider the configuration which is T-dual to the magnetic field solution along both $X^{1}$ and $X^{2}$ directions. The resulting configuration may correspond to a configuration of multiple D-particles on the torus. Such a system was studied in the case of a non-compact space [13]. In this case, the coordinates of the D-particles become noncommutative and the observable (4.11) represents the coupling of the D-particle to a symplectic form characterizing the noncommutativity. Therefore, D-particles with noncommutative coordinates may be described in terms of the string field theory in this dual background. Such a string field theory may be considered as another version of Matrix theories [14, 15] although it has no supersymmetry. It is also possible to make the relation between noncommutative geometry and constant magnetic field background manifest by a similarity transformation for string fields [16, 17]. It will be interesting to find such a similarity transformation in the background of the magnetic field solution. 


\section{Acknowledgments}

The authors would like to thank T. Erler, M. Kudrna, C. Maccaferri, M. Schnabl, Y. Kaneko and S. Watamura for helpful comments. We also thank the organizers of "SFT@HIT" at Holon, especially M. Kroyter, for hospitality. T. M. also would like to thank the organizers of "String Field Theory and String Phenomenology" at Harish-Chandra Research Institute, Allahabad, for their hospitality and for providing him a stimulating environment. This work was supported in part by JSPS Grant-in-Aid for Scientific Research (C) (JP25400242), JSPS Grant-in-Aid for Young Scientists (B) (JP25800134), and JSPS Grant-in-Aid for Scientific Research (C) (JP15K05056), and Nara Women's University Intramural Grant for Project Research. The research of T.M. was supported by the Grant Agency of the Czech Republic under the grant 17-22899S. The numerical computation in this work was partly carried out at the Yukawa Institute Computer Facility.

\section{A Conformal transformation of the vector vertex operator}

In this appendix, we would like to derive the conformal transformation (3.23) of the vector vertex operator. The OPE of the vertex operator with the energy momentum tensor is given by

$$
T(\zeta) \partial X^{\mu} e^{i \boldsymbol{p} \cdot \boldsymbol{X}}(\xi) \sim \frac{-i \alpha^{\prime} p^{\mu}}{(\zeta-\xi)^{3}} e^{i \boldsymbol{p} \cdot \boldsymbol{X}}(\xi)+\frac{\alpha^{\prime} \boldsymbol{p}^{2}+1}{(\zeta-\xi)^{2}} \partial X^{\mu} e^{i \boldsymbol{p} \cdot \boldsymbol{X}}(\xi)+\frac{1}{\zeta-\xi} \partial\left(\partial X^{\mu} e^{i \boldsymbol{p} \cdot \boldsymbol{X}}\right)(\xi) .
$$

The right hand side includes the tachyon vertex operator which is a primary field of weight $\alpha^{\prime} \boldsymbol{p}^{2}$ :

$$
T(\zeta) e^{i \boldsymbol{p} \cdot \boldsymbol{X}}(\xi) \sim \frac{\alpha^{\prime} \boldsymbol{p}^{2}}{(\zeta-\xi)^{2}} e^{i \boldsymbol{p} \cdot \boldsymbol{X}}(\xi)+\frac{1}{\zeta-\xi} \partial\left(e^{i \boldsymbol{p} \cdot \boldsymbol{X}}\right)(\xi) .
$$

Let us consider a conformal transformation $\xi \rightarrow z=f(\xi)$, under which a field $\phi(\xi)$ is transformed as

$$
\phi(\xi) \rightarrow f \circ \phi(\xi)=U_{f} \phi(\xi) U_{f}^{-1}
$$

Here, the operator $U_{f}$ is given in terms of the energy-momentum tensor as follows:

$$
U_{f}=e^{\mathcal{T}(v)}, \quad \mathcal{T}(v)=\oint \frac{d \xi}{2 \pi i} v(\xi) T(\xi), \quad f(\xi)=e^{v(\xi) \partial} \xi .
$$

Since the tachyon vertex is primary, it is transformed as

$$
U_{f} e^{i \boldsymbol{p} \cdot \boldsymbol{X}}(\xi) U_{f}^{-1}=\left(\frac{d z}{d \xi}\right)^{\alpha^{\prime} \boldsymbol{p}^{2}} e^{i \boldsymbol{p} \cdot \boldsymbol{X}}(z) .
$$

From the OPEs (A.1) and (A.2), we can derive the commutation relations of the vertex operators with $\mathcal{T}(v)$ :

$$
\begin{aligned}
{\left[\mathcal{T}(v), \partial X^{\mu} e^{i \boldsymbol{p} \cdot \boldsymbol{X}}(\xi)\right]=} & -\frac{i}{2} p^{\mu}\left(\partial^{2} v\right) e^{i \boldsymbol{p} \cdot \boldsymbol{X}}(\xi) \\
& +\left(\alpha^{\prime} \boldsymbol{p}^{2}+1\right)(\partial v) \partial X^{\mu} e^{i \boldsymbol{p} \cdot \boldsymbol{X}}(\xi)+v \partial\left(\partial X^{\mu} e^{i \boldsymbol{p} \cdot \boldsymbol{X}}\right)(\xi), \\
{\left[\mathcal{T}(v), e^{i \boldsymbol{p} \cdot \boldsymbol{X}}(\xi)\right]=} & \alpha^{\prime} \boldsymbol{p}^{2}(\partial v) e^{i \boldsymbol{p} \cdot \boldsymbol{X}}(\xi)+v \partial e^{i \boldsymbol{p} \cdot \boldsymbol{X}}(\xi) .
\end{aligned}
$$


Let us consider the transformation of the vector vertex operator under the following one parameter family of conformal transformations:

$$
e^{t \mathcal{T}(v)} \partial X^{\mu} e^{i \boldsymbol{p} \cdot \boldsymbol{X}}(\xi) e^{-t \mathcal{T}(v)} .
$$

By using the commutation relations (A.6) and (A.7), we can see that the result should be expressed as

$$
Y_{t}(\xi) \partial X^{\mu} e^{i \boldsymbol{p} \cdot \boldsymbol{X}}(\xi)+Z_{t}^{\mu}(\xi) e^{i \boldsymbol{p} \cdot \boldsymbol{X}}(\xi)
$$

where $Y_{t}(\xi)$ and $Z_{t}(\xi)$ are some functions of $t$ and $\xi$. Differentiating these with respect to $t$ and using (A.6) and (A.7), we obtain the equations

$$
\begin{aligned}
\frac{d Y_{t}(\xi)}{d t} & =\left(\alpha^{\prime} \boldsymbol{p}^{2}+1\right) v^{\prime}\left(f_{t}(\xi)\right) Y_{t}(\xi) \\
\frac{d Z_{t}^{\mu}(\xi)}{d t} & =\left(\alpha^{\prime} \boldsymbol{p}^{2}\right) v^{\prime}\left(f_{t}(\xi)\right) Z_{t}^{\mu}(\xi)-\frac{i}{2} \alpha^{\prime} p^{\mu} v^{\prime \prime}\left(f_{t}(\xi)\right) Y_{t}(\xi)
\end{aligned}
$$

where $f_{t}(\xi)$ is defined by $f_{t}(\xi)=\exp (t v(\xi) \partial) \xi$. Integrating these with the initial conditions $Y_{t=0}=1$ and $Z_{t=0}=0$, we find

$$
Y_{t}(\xi)=\left(f_{t}^{\prime}(\xi)\right)^{\alpha^{\prime} \boldsymbol{p}^{2}+1}, \quad Z_{t}^{\mu}(\xi)=-\frac{i}{2} p^{\mu} \frac{f_{t}^{\prime \prime}(\xi)}{f_{t}^{\prime}(\xi)}\left(f_{t}^{\prime}(\xi)\right)^{\alpha^{\prime} \boldsymbol{p}^{2}}
$$

By setting $t=1$ and $p^{\mu} \rightarrow-p^{\mu}$, we obtain the formula (3.23).

\section{B Calculation of correlation functions in the vector profile}

In this appendix, we will show how to calculate the correlation functions which appear in (3.21) and (3.22).

Here we begin with the calculation of the following 4-point functions on the complex $\zeta$ plane:

$$
\left\langle\partial X(\zeta) e^{-i \boldsymbol{p} \cdot \boldsymbol{X}}(\xi) \sigma_{*}^{k}\left(\xi_{1}\right) \bar{\sigma}_{*}^{l}\left(\xi_{2}\right)\right\rangle .
$$

Here $X=\left(X^{1}+i X^{2}\right) / \sqrt{2}$, and $\xi, \xi_{1}$ and $\xi_{2}$ are taken to be real and satisfy $\xi_{2}<\xi_{1}<\xi$, so that the operator $e^{-i \boldsymbol{p} \cdot \boldsymbol{X}}$ is on the boundary with the Neumann boundary condition. The correlation function is a 1 -form on the complex plane with respect to the variable $\zeta$ and it behaves as

$$
\sim \begin{cases}\left(\zeta-\xi_{1}\right)^{-\lambda} & \left(\zeta \sim \xi_{1}\right) \\ \left(\zeta-\xi_{2}\right)^{-1+\lambda} & \left(\zeta \sim \xi_{2}\right) \\ \zeta^{-2} & (\zeta \sim \infty)\end{cases}
$$

The first two are derived directly from boundary conditions of $X$. Moreover, in the limit $\zeta \sim \xi$, we find that the 4-point function behaves as

$$
=\frac{i \alpha^{\prime} p}{\zeta-\xi}\left\langle e^{-i \boldsymbol{p} \cdot \boldsymbol{X}}(\xi) \sigma_{*}^{k}\left(\xi_{1}\right) \bar{\sigma}_{*}^{l}\left(\xi_{2}\right)\right\rangle+\text { regular terms }
$$


which fixes the normalization of the four point function. These conditions determine the 4 -point function as

$$
\begin{aligned}
\left\langle\partial X(\zeta) e^{-i \boldsymbol{p} \cdot \boldsymbol{X}}(\xi) \sigma_{*}^{k}\left(\xi_{1}\right) \bar{\sigma}_{*}^{l}\left(\xi_{2}\right)\right\rangle= & i \alpha^{\prime} p \frac{\left(\zeta-\xi_{1}\right)^{-\lambda}\left(\zeta-\xi_{2}\right)^{-1+\lambda}\left(\xi-\xi_{1}\right)^{\lambda}\left(\xi-\xi_{2}\right)^{1-\lambda}}{\zeta-\xi} \\
& \times\left\langle e^{-i \boldsymbol{p} \cdot \boldsymbol{X}}(\xi) \sigma_{*}^{k}\left(\xi_{1}\right) \bar{\sigma}_{*}^{l}\left(\xi_{2}\right)\right\rangle
\end{aligned}
$$

where the 3-point function on the right hand side is given by (3.8) [2]. By taking the limit $\zeta \rightarrow \xi$ in (B.4), we obtain a correlation function involving the vector vertex operator on the complex plane:

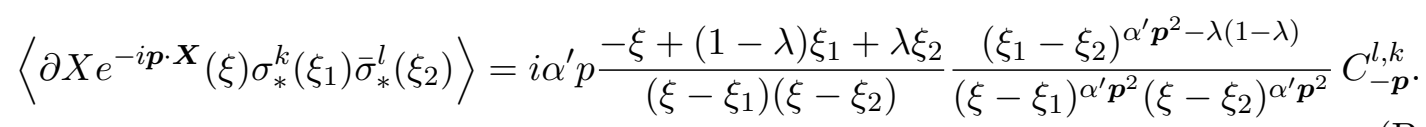

Taking the normalization of the modified BCC operators [2] into account, we obtain

$$
\begin{aligned}
& \left\langle\partial X e^{-i \boldsymbol{p} \cdot \boldsymbol{X}}(\xi) \sigma^{k}\left(\xi_{1}\right) \bar{\sigma}^{l}\left(\xi_{2}\right)\right\rangle
\end{aligned}
$$

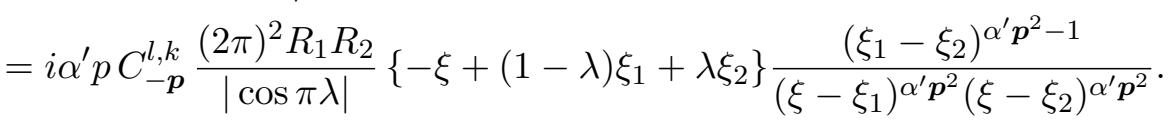

The correlation function for the conjugate vertex operator $\partial \tilde{X} e^{-i \boldsymbol{p} \cdot \boldsymbol{X}}\left(\tilde{X}=\left(X^{1}-i X^{2}\right) / \sqrt{2}\right)$ can be calculated in a similar way and we find

$$
\begin{aligned}
& \left\langle\partial \tilde{X} e^{-i \boldsymbol{p} \cdot \boldsymbol{X}}(\xi) \sigma^{k}\left(\xi_{1}\right) \bar{\sigma}^{l}\left(\xi_{2}\right)\right\rangle
\end{aligned}
$$

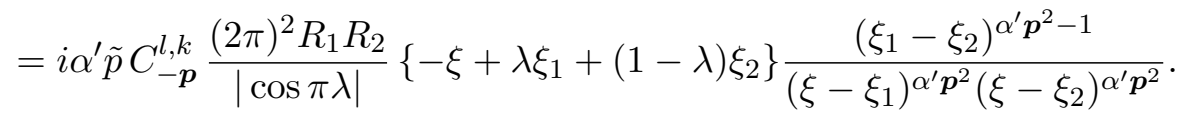

Eq. (B.7) can also be obtained by using the fact that the $X^{2}$ parity transformation $X^{2} \rightarrow$ $-X^{2}$ corresponds to the following transformations of the parameters:[2]

$$
p \rightarrow \tilde{p}, \quad \lambda \rightarrow 1-\lambda .
$$

Having found the 3-point functions on the complex plane, we can find the one on the infinite cylinder with circumference $L$ by the conformal transformation:

$$
z=\frac{L}{\pi} \arctan \xi
$$

By using the transformation law (3.23), we obtain the following 3-point functions on the cylinder from (B.6) and (B.7):

$$
\begin{aligned}
&\left\langle\partial X^{\mu} e^{-i \boldsymbol{p} \cdot \boldsymbol{X}}\left(z_{1}\right) \sigma^{k}\left(z_{2}\right) \bar{\sigma}^{l}\left(z_{3}\right)\right\rangle_{L} \\
&=\left(\frac{\pi}{L}\right)^{h} C_{-p}^{l, k} \frac{(2 \pi)^{2} R_{1} R_{2}}{|\cos \pi \lambda|} \frac{\left\{\sin \frac{\pi\left(z_{2}-z_{3}\right)}{L}\right\}^{h-1}}{\left\{\sin \frac{\pi\left(z_{1}-z_{2}\right)}{L} \sin \frac{\pi\left(z_{1}-z_{3}\right)}{L}\right\}^{h}} \\
& \times {\left[\left(\lambda-\frac{1}{2}\right) \alpha^{\prime} \epsilon^{\mu \nu} p_{\nu} \sin \frac{\pi\left(z_{2}-z_{3}\right)}{L}\right.} \\
&\left.\quad-\frac{i}{2} \alpha^{\prime} p^{\mu}\left\{\sin \frac{\pi\left(z_{1}-z_{2}\right)}{L} \cos \frac{\pi\left(z_{1}-z_{3}\right)}{L}+\sin \frac{\pi\left(z_{1}-z_{3}\right)}{L} \cos \frac{\pi\left(z_{1}-z_{2}\right)}{L}\right\}\right],
\end{aligned}
$$

where $h=\alpha^{\prime} \boldsymbol{p}^{2}+1$ and $\epsilon^{12}=-\epsilon^{21}=1$. 
The matter correlation functions that appear in (3.21) are calculated by using (B.9) as

$$
\begin{aligned}
&\left\langle\partial X^{\mu} e^{-i \boldsymbol{p} \cdot \boldsymbol{X}}\left(L-\frac{1}{2}\right) \partial \sigma^{k}\left(t_{1}+t_{2}\right) \bar{\sigma}^{l}\left(t_{1}\right)\right\rangle_{L} \\
&=\left(\frac{\pi}{L}\right)^{h+1} C_{-\boldsymbol{p}}^{l, k} \frac{(2 \pi)^{2} R_{1} R_{2}}{|\cos \pi \lambda|}\left[\left(\lambda-\frac{1}{2}\right) \alpha^{\prime} \epsilon^{\mu \nu} p_{\nu} h \frac{\sin ^{h-1} \theta_{t_{2}}}{\sin ^{h} \theta_{t_{1}+\frac{1}{2}} \sin ^{h+1} \theta_{t_{3}+\frac{1}{2}}}\right. \\
& \times\left(\cos \theta_{t_{2}} \sin \theta_{t_{3}+\frac{1}{2}}+\sin \theta_{t_{2}} \cos \theta_{t_{3}+\frac{1}{2}}\right) \\
&-\frac{i}{2} \alpha^{\prime} p^{\mu} \frac{\sin ^{h-2} \theta_{t_{2}}}{\sin ^{h} \theta_{t_{1}+\frac{1}{2}} \sin ^{h+1} \theta_{t_{3}+\frac{1}{2}}}\left\{-(h-1) \cos \theta_{t_{2}} \cos \theta_{t_{1}+\frac{1}{2}} \sin ^{2} \theta_{t_{3}+\frac{1}{2}}\right. \\
& \quad+(h-1) \cos \theta_{t_{2}} \sin \theta_{t_{1}+\frac{1}{2}} \sin \theta_{t_{3}+\frac{1}{2}} \cos \theta_{t_{3}+\frac{1}{2}} \\
&-(h-1) \sin \theta_{t_{2}} \cos \theta_{t_{1}+\frac{1}{2}} \sin \theta_{t_{3}+\frac{1}{2}} \cos \theta_{t_{3}+\frac{1}{2}} \\
&\left.\left.+h \sin \theta_{t_{2}} \sin \theta_{t_{1}+\frac{1}{2}} \cos ^{2} \theta_{t_{3}+\frac{1}{2}}+\sin \theta_{t_{2}} \sin \theta_{t_{1}+\frac{1}{2}} \sin ^{2} \theta_{t_{3}+\frac{1}{2}}\right\}\right],
\end{aligned}
$$

and

$$
\begin{aligned}
&\left\langle\partial X^{\mu} e^{-i \boldsymbol{p} \cdot \boldsymbol{X}}\left(L-\frac{1}{2}\right) \sigma^{k}\left(t_{1}+t_{2}\right) \partial \bar{\sigma}^{l}\left(t_{1}\right)\right\rangle_{L} \\
&=\left(\frac{\pi}{L}\right)^{h+1} C_{-\boldsymbol{p}}^{l, k} \frac{(2 \pi)^{2} R_{1} R_{2}}{|\cos \pi \lambda|}\left[-\left(\lambda-\frac{1}{2}\right) \alpha^{\prime} \epsilon^{\mu \nu} p_{\nu} h \frac{\sin ^{h-1} \theta_{t_{2}}}{\sin ^{h+1} \theta_{t_{1}+\frac{1}{2}} \sin ^{h} \theta_{t_{3}+\frac{1}{2}}}\right. \\
& \quad \times\left(\cos \theta_{t_{2}} \sin \theta_{t_{1}+\frac{1}{2}}+\sin \theta_{t_{2}} \cos \theta_{t_{1}+\frac{1}{2}}\right) \\
&-\frac{i}{2} \alpha^{\prime} p^{\mu} \frac{\sin ^{h-2} \theta_{t_{2}}}{\sin ^{h+1} \theta_{t_{1}+\frac{1}{2}} \sin ^{h} \theta_{t_{3}+\frac{1}{2}}}\left\{-(h-1) \cos \theta_{t_{2}} \cos \theta_{t_{3}+\frac{1}{2}} \sin ^{2} \theta_{t_{1}+\frac{1}{2}}\right. \\
&+(h-1) \cos \theta_{t_{2}} \sin \theta_{t_{3}+\frac{1}{2}} \sin \theta_{t_{1}+\frac{1}{2}} \cos \theta_{t_{1}+\frac{1}{2}} \\
& \quad-(h-1) \sin \theta_{t_{2}} \cos \theta_{t_{3}+\frac{1}{2}} \sin \theta_{t_{1}+\frac{1}{2}} \cos \theta_{t_{1}+\frac{1}{2}} \\
&\left.\left.+h \sin \theta_{t_{2}} \sin \theta_{t_{3}+\frac{1}{2}} \cos ^{2} \theta_{t_{1}+\frac{1}{2}}+\sin \theta_{t_{2}} \sin \theta_{t_{3}+\frac{1}{2}} \sin ^{2} \theta_{t_{1}+\frac{1}{2}}\right\}\right]
\end{aligned}
$$

where $\theta_{s}$ is defined by $\theta_{s}=\pi s / L$. The ghost correlation functions which appear in (3.21) are given as

$$
\begin{aligned}
\left\langle c \partial c\left(L-\frac{1}{2}\right) c\left(t_{1}+t_{2}\right)\right\rangle_{L} & =-\left(\frac{L}{\pi}\right)^{2} \sin ^{2} \theta_{t_{3}+\frac{1}{2}} \\
\left\langle c \partial c\left(L-\frac{1}{2}\right) c\left(t_{1}\right)\right\rangle_{L} & =-\left(\frac{L}{\pi}\right)^{2} \sin ^{2} \theta_{t_{1}+\frac{1}{2}}
\end{aligned}
$$

Combining these results, we find that the integrand in (3.21) turns out to be

$$
\begin{array}{r}
\left\langle\partial X^{\mu} e^{-i \boldsymbol{p} \cdot \boldsymbol{X}}\left(L-\frac{1}{2}\right) \partial \sigma^{k}\left(t_{1}+t_{2}\right) \bar{\sigma}^{l}\left(t_{1}\right)\right\rangle_{L}\left\langle c \partial c\left(L-\frac{1}{2}\right) c\left(t_{1}+t_{2}\right)\right\rangle_{L} \\
-\left\langle\partial X^{\mu} e^{-i \boldsymbol{p} \cdot \boldsymbol{X}}\left(L-\frac{1}{2}\right) \sigma^{k}\left(t_{1}+t_{2}\right) \partial \bar{\sigma}^{l}\left(t_{1}\right)\right\rangle_{L}\left\langle c \partial c\left(L-\frac{1}{2}\right) c\left(t_{1}\right)\right\rangle_{L}
\end{array}
$$




$$
\begin{aligned}
=\left(\frac{\pi}{L}\right)^{h-1} & C_{-p}^{l, k} \frac{(2 \pi)^{2} R_{1} R_{2}}{|\cos \pi \lambda|}\left[-\left(\lambda-\frac{1}{2}\right) \alpha^{\prime} \epsilon^{\mu \nu} p_{\nu} h \frac{\sin ^{h-1} \theta_{t_{2}}}{\sin ^{h} \theta_{t_{1}+\frac{1}{2}} \sin ^{h} \theta_{t_{3}+\frac{1}{2}}} \times\right. \\
& \times\left\{\cos \theta_{t_{2}}\left(\sin ^{2} \theta_{t_{1}+\frac{1}{2}}+\sin ^{2} \theta_{t_{3}+\frac{1}{2}}\right)+\frac{1}{2} \sin \theta_{t_{2}}\left(\sin 2 \theta_{t_{1}+\frac{1}{2}}+\sin 2 \theta_{t_{3}+\frac{1}{2}}\right)\right\} \\
+\frac{i}{2} \alpha^{\prime} p^{\mu} & \frac{\sin ^{h-2} \theta_{t_{2}}}{\sin ^{h} \theta_{t_{1}+\frac{1}{2}} \sin ^{h} \theta_{t_{3}+\frac{1}{2}}} \\
& \times\left\{(h-1) \cos \theta_{t_{2}}\left(\sin ^{3} \theta_{t_{1}+\frac{1}{2}} \cos \theta_{t_{3}+\frac{1}{2}}-\sin ^{3} \theta_{t_{3}+\frac{1}{2}} \cos \theta_{t_{1}+\frac{1}{2}}\right)\right. \\
& -(h-1) \cos \theta_{t_{2}} \sin \theta_{t_{1}+\frac{1}{2}} \sin \theta_{t_{3}+\frac{1}{2}}\left(\sin \theta_{t_{1}+\frac{1}{2}} \cos \theta_{t_{1}+\frac{1}{2}}-\sin \theta_{t_{3}+\frac{1}{2}} \cos \theta_{t_{3}+\frac{1}{2}}\right) \\
& +(h-1) \sin \theta_{t_{2}} \cos \theta_{t_{1}+\frac{1}{2}} \cos \theta_{t_{3}+\frac{1}{2}}\left(\sin ^{2} \theta_{t_{1}+\frac{1}{2}}-\sin ^{2} \theta_{t_{3}+\frac{1}{2}}\right) \\
& -h \sin \theta_{t_{2}} \sin \theta_{t_{1}+\frac{1}{2}} \sin \theta_{t_{3}+\frac{1}{2}}\left(\cos ^{2} \theta_{t_{1}+\frac{1}{2}}-\cos ^{2} \theta_{t_{3}+\frac{1}{2}}\right) \\
& \left.\left.-\sin \theta_{t_{2}} \sin \theta_{t_{1}+\frac{1}{2}} \sin \theta_{t_{3}+\frac{1}{2}}\left(\sin ^{2} \theta_{t_{1}+\frac{1}{2}}-\sin ^{2} \theta_{t_{3}+\frac{1}{2}}\right)\right\}\right] .
\end{aligned}
$$

The second term in the bracket on the right hand side proportional to $p^{\mu}$ is antisymmetric under the interchange of $t_{1}$ and $t_{3}$. Since the integration measure in (3.21) is symmetric with respect to it, the second term does not contribute to the vector profile.

Next let us turn to (3.22). The correlation function which appears in the integrand in $(3.22)$ is given as

$$
\begin{aligned}
\left(\frac{\pi}{L}\right)^{h-1} & (h-1) C_{-p}^{l, k} \frac{(2 \pi)^{2} R_{1} R_{2}}{|\cos \pi \lambda|} \frac{\sin ^{h-2} \theta_{t_{2}}}{\sin ^{h} \theta_{t_{1}+\frac{1}{2}} \sin ^{h} \theta_{t_{3}+\frac{1}{2}}} \\
\times \frac{1}{L}[ & \frac{\pi}{L} \sin \theta_{t_{2}}\left\{\left(1+2 t_{1}\right) \cos \theta_{t_{1}+\frac{1}{2}} \sin \theta_{t_{3}+\frac{1}{2}}-\left(1+2 t_{3}\right) \cos \theta_{t_{3}+\frac{1}{2}} \sin \theta_{t_{1}+\frac{1}{2}}\right\} \\
& +\frac{2 \pi\left(t_{1}-t_{3}\right)}{L} \cos \theta_{t_{2}} \sin \theta_{t_{1}+\frac{1}{2}} \sin \theta_{t_{3}+\frac{1}{2}} \\
& -2 \sin \theta_{t_{2}} \sin \theta_{t_{1}+\frac{1}{2}} \sin \theta_{t_{3}+\frac{1}{2}}\left(\cos ^{2} \theta_{t_{1}+\frac{1}{2}}-\cos ^{2} \theta_{t_{3}+\frac{1}{2}}\right) \\
& \left.-2 \cos \theta_{t_{2}}\left(\cos \theta_{t_{1}+\frac{1}{2}} \sin ^{2} \theta_{t_{1}+\frac{1}{2}} \sin \theta_{t_{3}+\frac{1}{2}}-\cos \theta_{t_{3}+\frac{1}{2}} \sin ^{2} \theta_{t_{3}+\frac{1}{2}} \sin \theta_{t_{1}+\frac{1}{2}}\right)\right] .
\end{aligned}
$$

Since (B.15) is antisymmetric under the interchange of $t_{1}$ and $t_{3}$, we find that the right hand side of (3.22) vanishes.

Open Access. This article is distributed under the terms of the Creative Commons Attribution License (CC-BY 4.0), which permits any use, distribution and reproduction in any medium, provided the original author(s) and source are credited.

\section{References}

[1] T. Erler and C. Maccaferri, String field theory solution for any open string background, JHEP 10 (2014) 029 [arXiv: 1406.3021] [INSPIRE]. 
[2] N. Ishibashi, I. Kishimoto and T. Takahashi, String field theory solution corresponding to constant background magnetic field, PTEP 2017 (2017) 013B06 [arXiv:1610.05911] [INSPIRE].

[3] B. Zwiebach, Interpolating string field theories, Mod. Phys. Lett. A 7 (1992) 1079 [hep-th/9202015] [INSPIRE].

[4] T. Erler and M. Schnabl, A simple analytic solution for tachyon condensation, JHEP 10 (2009) 066 [arXiv:0906. 0979] [INSPIRE].

[5] A. Abouelsaood, C.G. Callan Jr., C.R. Nappi and S.A. Yost, Open strings in background gauge fields, Nucl. Phys. B 280 (1987) 599 [inSPIRE].

[6] G.T. Horowitz and A. Strominger, Translations as inner derivations and associativity anomalies in open string field theory, Phys. Lett. B 185 (1987) 45 [INSPIRE].

[7] T. Kawano, I. Kishimoto and T. Takahashi, Gauge invariant overlaps for classical solutions in open string field theory, Nucl. Phys. B 803 (2008) 135 [arXiv:0804.1541] [INSPIRE].

[8] J. Polchinski, String theory. Volume 1: an introduction to the bosonic string, Cambridge Monographs on Mathematical Physics, Cambridge UNiversity Press, Cambridge U.K. (1998).

[9] H. Grosse, C. Klimčík and P. Prešnajder, Topologically nontrivial field configurations in noncommutative geometry, Commun. Math. Phys. 178 (1996) 507 [hep-th/9510083] [INSPIRE].

[10] A. Connes, M.R. Douglas and A.S. Schwarz, Noncommutative geometry and matrix theory: compactification on tori, JHEP 02 (1998) 003 [hep-th/9711162] [INSPIRE].

[11] E. Witten, Overview of k-theory applied to strings, Int. J. Mod. Phys. A 16 (2001) 693 [hep-th/0007175] [INSPIRE].

[12] U. Carow-Watamura, H. Steinacker and S. Watamura, Monopole bundles over fuzzy complex projective spaces, J. Geom. Phys. 54 (2005) 373 [hep-th/0404130] [INSPIRE].

[13] N. Ishibashi, p-branes from ( $p-2)$-branes in the bosonic string theory, Nucl. Phys. B 539 (1999) 107 [hep-th/9804163] [INSPIRE].

[14] T. Banks, W. Fischler, S.H. Shenker and L. Susskind, $M$ theory as a matrix model: a conjecture, Phys. Rev. D 55 (1997) 5112 [hep-th/9610043] [INSPIRE].

[15] N. Ishibashi, H. Kawai, Y. Kitazawa and A. Tsuchiya, A large $N$ reduced model as superstring, Nucl. Phys. B 498 (1997) 467 [hep-th/9612115] [InSPIRE].

[16] F. Sugino, Witten's open string field theory in constant B field background, JHEP 03 (2000) 017 [hep-th/9912254] [INSPIRE].

[17] T. Kawano and T. Takahashi, Open string field theory on noncommutative space, Prog. Theor. Phys. 104 (2000) 459 [hep-th/9912274] [inSPIRE]. 